\title{
Multiphase ISM in low luminosity radio galaxies: A case study of NGC 708
}

\author{
Sahu, Sheetal $\mathbf{K}^{1}$; Pandey, $\mathbf{S} \mathbf{K}^{1}$; Chaware, $\mathbf{L}^{1}$; Pandge, $\mathbf{M} \mathbf{B}^{2,3}$ \\ ${ }^{1}$ SOS in Physics and Astrophysics, Pt. Ravishankar Shukla University, Raipur, India 492010 \\ ${ }^{2}$ Mahatma Basweshswar Mahavidyalaya, Latur, Maharastra, India 413512. \\ ${ }^{3}$ Dayanand Science College, Latur, Maharastra India.413512. \\ email: sheetal.phy14@gmail.com; proskp@gmail.com; chaware.laxmikant@gmail.com
}

\begin{abstract}
We present a multi-wavelength study of a nearby radio loud elliptical galaxy NGC 708 , selected from the Bologna B2 sample of radio galaxies. We obtained optical broad band and narrow images from IGO $2 \mathrm{~m}$ telescope (Pune, India). We supplement the multi-wavelength coverage of the observation by using X-ray data from Chandra, infrared data from 2MASS, Spitzer and WISE and optical image from DSS and HST. In order to investigate properties of interstellar medium, we have generated unsharp-masked, color, residual, quotient, dust extinction, $\mathrm{H} \alpha$ emission maps. From the derived maps it is evident that cool gas, dust, warm ionized $\mathrm{H} \alpha$ and hot X-ray gas are spatially associated with each other. We investigate the inner and outer photometric and kinematic properties of the galaxy using surface brightness profiles. From $\mathrm{X}$-ray $2 \mathrm{~d}$ beta model, unsharp masking, surface brightness profiles techniques, it is evident that pair of X-ray cavities are present in this system and which are $\sim 5.6 \mathrm{Kpc}$ away from the central $\mathrm{X}$-ray source.
\end{abstract}

Keywords: Galaxies: early type, surface photometry, ISM, Cooling flows, AGN feedback.

\section{Introduction, Observation and Analysis}

This paper reports multiband study of NGC 708 a radio loud elliptical galaxy selected from B2 sample ${ }^{[1][3]}$ for which observational data in Radio, NIR, UV and X-ray band are already available through various data archives. The main goal of this multi wavelength study is to investigate possible inter-correlation among different constituents of ISM and coexistence of multiphase ISM in extra-galactic environment. This work adresses a wide variety of issues related to different physical properties of dust in early type galaxies. In particular, the data is used to (1) perform multi-band surface photometry and generate composite and multicolor profiles for the galaxies. (2) use color profiles to study morphology of molecular gas, dust and other faint features. (3) obtain dust properties from wavelength-dependent dust extinction and compare it with the properties of dust in the Milky-way. (4) compare the spatial distribution of the gas and dust revealed in diffirent wavelength bands. (5) study morphology and properties of ionized gas and star forming regions detected in the $\mathrm{H} \alpha$ narrow band images.

The photometric observations were carried out during June 14-17, 2012 using IGO two meter optical telescope at IUCAA, Pune (India). The integrated exposure time in B, V, R and $\mathrm{H} \alpha$ bands were 3600 s, 2700s, 1800s, 5400s respectively. Typical values of seeing during our observations were in range of $\sim 1.5$. Standard techniques were applied to process the data as described in [4][6]. The radial profiles of surface brightness $(\mu)$, position angle (PA), ellipticity (e) and $B_{4}$ (boxiness/ diskyness) parameters were generated in images of all the wavebands using the 'ellipse' task available in $I R A F^{[4]}$.

\section{Results and Conclusions}

(1) Radial profiles of surface brightness $(\mu)$, position angle (PA), ellipticity (e) and $B_{4}$, indicates peculiar feature within its central 10 arcsec region with bump in the surface brightness profile near at 2-4 arcsec. (2) The optical colors (i.e. B-V, V-R, B-R) in conjunction with the Infrared color maps, indicates existence of cool gas surrounding the nuclear dust lane in the central region of the galaxy. (3) The derived extinction curve for NGC 708 shows significant difference at the $\mathrm{R}$ band, as compared to the extinction curve of the Milky-way. (4) The estimated value of $R_{V}$ for NGC 708 is $6.0 \pm 1.2$, which is considerbly higher than the canonical value of $R_{V}$ 
Table 1. Basic Information, Dust Extinction and Other Properties.

\begin{tabular}{|c|c|c|c|c|c|}
\hline Object name & | NGC 708 & Extinct. Coef. & $R_{B} \pm \Delta R_{B}$ & $R_{V} \pm \Delta R_{V}$ & $R_{R} \pm \Delta R_{R}$ \\
\hline RA (hh:mm:ss) & $01: 52: 46.48$ & \multirow{2}{*}{$\begin{array}{l}\text { Milky Way } \\
\text { NGC } 708\end{array}$} & \multirow{2}{*}{$\begin{array}{l}4.1 \\
7.0 \pm 1.2\end{array}$} & \multirow{2}{*}{$\begin{array}{l}3.1 \\
6.0 \pm 1.2\end{array}$} & \multirow{2}{*}{$\begin{array}{l}2.3 \\
7.3 \pm 1.2 \\
\end{array}$} \\
\hline DEC (dd:mm:ss) & $\mid+36: 09: 06.6$ & & & & \\
\hline Dust Mass $M_{d(I R A S)}$ & $\left|5.75 \pm 0.03\left(M_{\odot}\right)\right|$ & \multirow{2}{*}{$\begin{array}{l}\text { Molecular gas } \\
\text { mass } M_{H 2}\end{array}$} & \multirow{2}{*}{$\mid \begin{array}{l}8.6 \pm 0.2 \\
\left(M_{\odot}\right)\end{array}$} & & \\
\hline$\overline{\text { Dust Temp. } T_{d}}$ & $30.39 \pm 5.7(\mathrm{~K})$ & & & & \\
\hline$\overline{S F R_{F I R}}$ & $11.87\left(M_{\odot} / \mathrm{Yr}\right)$ & $S F R_{H \alpha}$ & $1.75\left(M_{\odot} / \mathrm{Yr}\right)$ & & \\
\hline
\end{tabular}
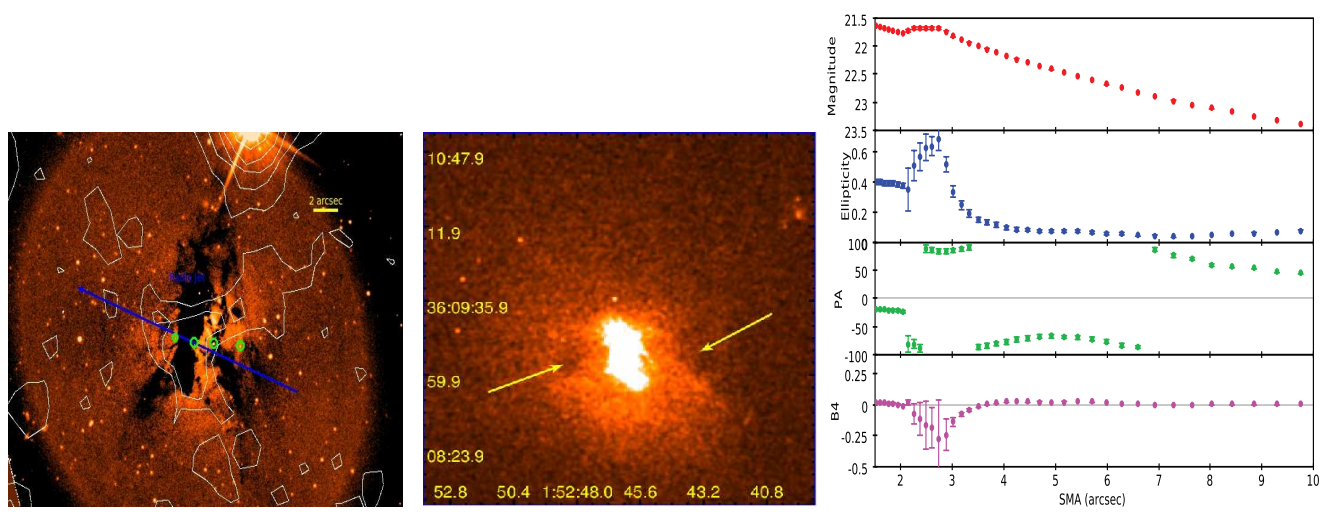

Figure 1. Left: Residual map HST [F814W] showing correlation among dust with radio jet(blue),

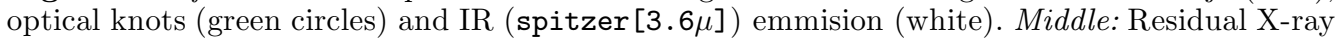
$\left(0.3-7.0 \mathrm{keV} 3^{\prime} \times 3\right.$ ' Chandra) image, reveal substructures in the central region. Right: Radial profiles of surface brightness $(\mu)$, position angle (PA), ellipticity (e) and $B_{4}$, in V band (IGO) within 10 arcsec with bumpy region.

(3.1) for Milky-way. (5) We identified three optical knots (two are located in the east and one is in the west from the center, extended within central $3 \mathrm{kpc}$ region of the galaxy) in HST images and it looks a jet like feature in IGO V-band image. (6) Distribution of ionized gas in NGC 708 revealed by $\mathrm{H} \alpha$ emission map is interesting, namely, an angular $\mathrm{V}$ shaped morphology having spiral arm like feature within its central $3 \mathrm{kpc}$ region. (7) The multi-band imaging analysis of NGC 708 reveals a qualitative spatial correspondence among the morphologies of the dust, $\mathrm{H} \alpha$, $\mathrm{IR}$, and radio emissions as well as diffuse X-ray emission. This in turn implies co-existence of multiphase ISM and their physical association in the sample galaxy ${ }^{[2][5]}$. (8) The diffuse X-ray emission and residual maps confirm the detection of X-ray cavity pair ${ }^{[7]}$ as has been found in previous studies.

\section{References}

[1] Cappetti A, et al, 2000, A\&A, 362, 871C. [2] Finkelman I., et al, 2010, MNRAS, 409, 727F. [3] Gonzalez-Serrano J.I., et al., 2000, A\&AS, 461, 103. [4] Jedrzejewski R.I., 1987, MNRAS, 226, 747. [5] Patil M. K., Pandey S.K., et al, 2007,A\&A, 461,103. [6] Sahu, D. K., Pandey S. K.,et al 1998, A\&A, 333, 803. [7] Pandge M B et al.2013, A\&SS, 345, 183-193. 